\title{
Investigation of left ventricular function by digital subtraction angiography
}

\author{
R A GREENBAUM, T R EVANS \\ From the London Imaging Centre, and the Royal Free Hospital, London
}

SUMMARY Twenty five patients who had undergone conventional coronary arteriography and left ventriculography for suspected coronary artery disease had left ventriculography performed by digital subtraction angiography as outpatients; there were no complications requiring admission to hospital. A catheter was introduced percutaneously and positioned in the superior vena cava; 40-50 $\mathrm{ml}$ of contrast medium was injected at $16-18 \mathrm{ml} / \mathrm{s}$. Left ventriculograms were recorded in the $30^{\circ}$ right anterior oblique projection. Diagnostic images were recorded in each case. Left ventricular wall movement was assessed as normal in nine patients, globally hypokinetic in 10, and aneurysmal in six. In each case there was agreement between the two methods. Ejection fraction varied between $17 \%$ and $78 \%$. It is concluded that digital subtraction angiography provides a reliable assessment of overall left ventricular function and distinguishes between those patients with a potentially resectable lesion and those with global impairment of function.

After intravenous injection of a contrast medium the concentration attained in the left ventricle and systemic arteries is too low to produce reliable imaging with conventional $x$ ray techniques. For precise characterisation of left ventricular function current practice relies largely on arterial puncture and selective cardiac catheterisation. This often requires admission to hospital. Advances in computer technology now permit radiographic examination of the left ventricle and major arteries after an intravenous bolus injection of a contrast medium. ${ }^{1}$

Many patients are investigated for dyspnoea that is thought to be due to left ventricular dysfunction. They are usually subdivided into those with a surgically correctable lesion, such as a left ventricular aneurysm, and those whose condition cannot be improved by surgery because of generalised left ventricular impairment. At present the most reliable means of distinguishing between these groups of patients is by direct left ventriculography. The purpose of this study was to show that digital subtraction angiography can provide diagnostic images of the left ventricle and that it enables those patients with a potentially surgically remediable lesion to be selected.

Requests for reprints to Dr R A Greenbaum, Department of Cardiology, Royal Free Hospital, London NW3 2QG.

Accepted for publication 4 October 1983
Patients and methods

Twenty five patients (18 men and seven women, mean age 53.3 (range 39.8-67.1) years) were investigated using a Technicare DS-960 digital subtraction angiographic system. Each patient had been previously investigated by conventional coronary arteriography and left ventriculography. The mean interval between the two investigations was 13 weeks (range $0.5-28$ weeks). The patients were investigated as outpatients after an overnight fast. A purpose designed catheter (Cordis or Cooks), size $5 \mathrm{~F}$ or $6 \mathrm{~F}$, was introduced percutaneously into a superficial vein in the antecubital fossa. In two cases in which this was not possible a femoral approach was successfully used. When feasible the tip of the catheter was positioned in the superior vena cava. In some instances the tip would not advance beyond the cephalic vein, and this was accepted. The contrast medium used was Cardio-Conray (May and Baker) in a dose of 0.5-0.6 $\mathrm{ml} / \mathrm{kg}$. This was delivered using a Medrad power injector at a rate of 16-18 ml/s. Images were acquired at 6 frames/s using a $256 \times 256$ pixel density and a 15 $\mathrm{cm}$ or $22 \mathrm{~cm}$ field. A $30^{\circ}$ right anterior oblique projection was used in each case. End systolic and end diastolic frames were selected as those with the smallest and largest areas. A copy of each was taken using a video imager (Matrix Instruments). Ejection fraction was calculated using a Numonics $123 \mathrm{~B}$ clinical 
graphics analyser programmed for the Dodge equation. ${ }^{2}$ Using the same graphics analyser ejection fraction was calculated from appropriate frames taken from the conventional angiogram.

\section{PRINCIPLE OF DIGITAL SUBTRACTION ANGIOGRAPHY}

In the Technicare system $x$ rays are generated in the conventional manner and having passed through the patient enter the image intensifier (Fig. 1). The signal from a television camera focused on the output phosphor of the image intensifier is fed to the analogue to digital converter. The acquisition time of the camera may be varied to allow different frame rates. Once in digital form there is no further possibility of noise degrading the signal quality. The signal is converted back to analogue form only for the purposes of display. The core of the system is a DEC PDP 11/34 computer. The digitised data for each frame are stored on disk. For more permanent storage the data are transferred on to magnetic tape. When data are acquired one frame recorded before injection of contrast, the mask frame, is stored in one of the system's memories. The mask frame contains soft tissue and bony shadows. After the injection of a contrast medium a composite frame containing both background and contrast is acquired in another of the system's memories. The mask frame is then subtracted from the composite image. The computer allows this to be achieved virtually instantaneously. The subtraction process is then repeated for each composite frame as it is acquired. The resultant images show vascular structures containing contrast medium with back- ground skeletal and soft tissue structures largely removed. To obtain the best possible subtraction image it is imperative for the patient to remain completely still while the images are recorded. The image chosen as the mask may be varied so that the optimal result is obtained. The system allows selection of field size, frame rate, and pixel density.

\section{PROCESSING OF ACQUIRED DATA}

Because the images are stored in digital form there is a wide range of possibilities for processing the data after its acquisition. For clinical purposes a hard copy of left ventricular outline is produced in an $x$ ray type format. A video display, however, provides a much better appreciation of left ventricular wall movement. Ejection fraction can be calculated by the computer, and if a suitable calibration is provided stroke volume may be estimated. It is in the techniques used after processing that many of the future refinements of this system will be made. A limitation of the method is a degree of uncertainty concerning the precise location of the aortic valve. There are frames, acquired after contrast has cleared from the left atrium, that allow sufficient definition of the left ventricular outflow tract for a satisfactory estimate to be made.

\section{Results}

All the patients were studied to investigate left ventricular wall motion as seen in the right anterior oblique projection. Ten of the patients had globally hypokinetic left ventricular function, six a discrete

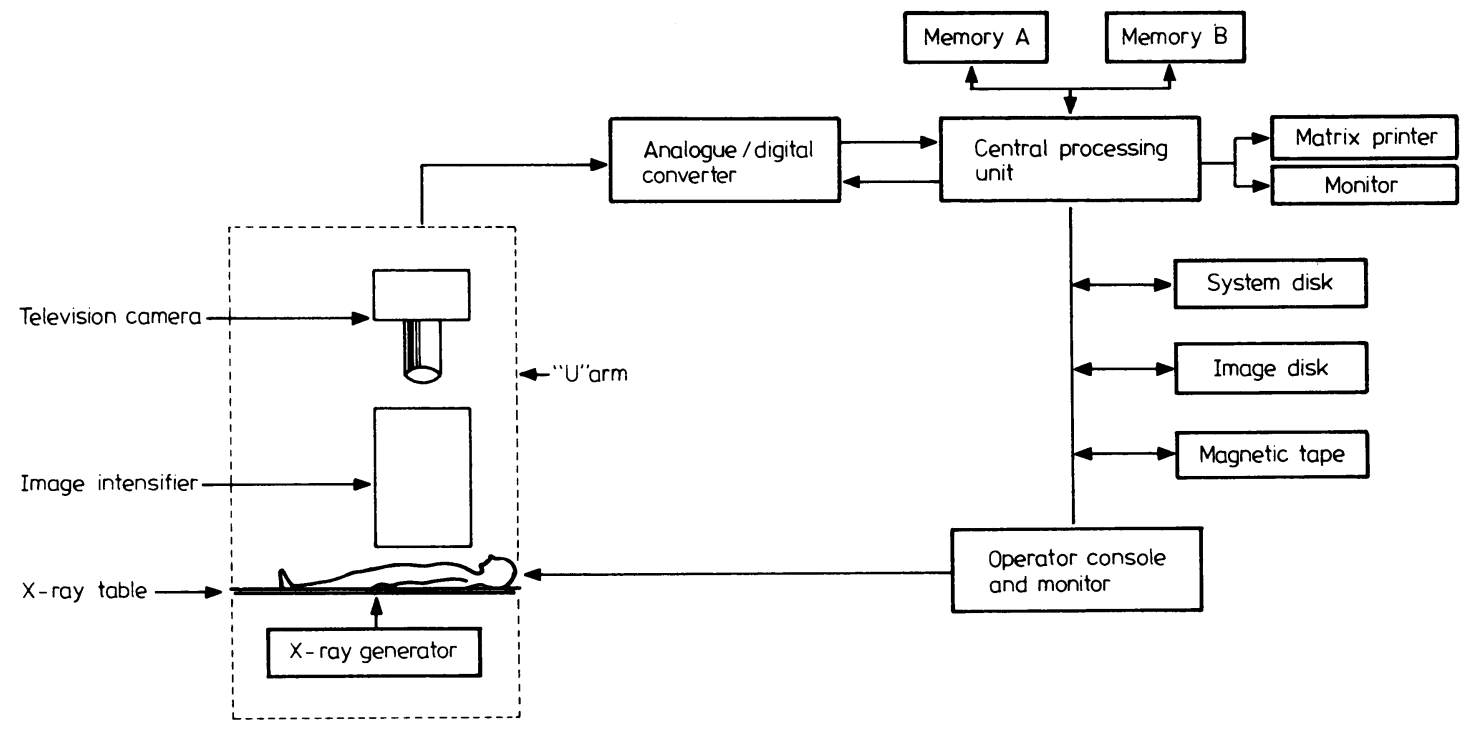

Fig. 1 Block diagram of the major components of the Technicare DS-960 system used for digital subtraction angiography. 
aneurysm, and nine normal left ventricular function. In each case overall left ventricular wall movement, as visualised by the two methods, was independently assessed. There was agreement in each case on the overall assessment of left ventricular function. Fig. 2 shows the relation between the values of ejection fraction calculated by each method; the results of 20 patients are plotted. In the remaining five cases no comparison was possible because of the frequency of extrasystoles in the conventional angiogram. Fig. 3 shows examples of digital images of normal and globally hypokinetic left ventricles and a left ventricular aneurysm at end diastole and end systole; it was not possible to identify the coronary arteries.

\section{Discussion}

Attempts to visualise the left ventricle after intravenous injection of a contrast medium using conventional $x$ ray equipment ${ }^{3}$ have proved impractical. The development of computer based techniques of image processing have led to renewed attempts to visualise the left ventricle. Kruger $e t \mathrm{al}^{4}$ demonstrated the left ventricle in dogs and a human volunteer after an intravenous injection of contrast. The practicality of the intravenous technique and the value of various projections were shown using research apparatus in seven patients with normal hearts. ${ }^{5} \mathrm{~A}$ prototype system has been described, ${ }^{6}$ which emphasises the importance of defining left ventricular function and suggests imaging of the entire coronary artery tree as an objective for this technique. Good correlation has been reported for patients with ejection fractions greater than 0.55 calculated from digital and conventional angiograms. ${ }^{78}$

Other groups of workers have developed the alter-

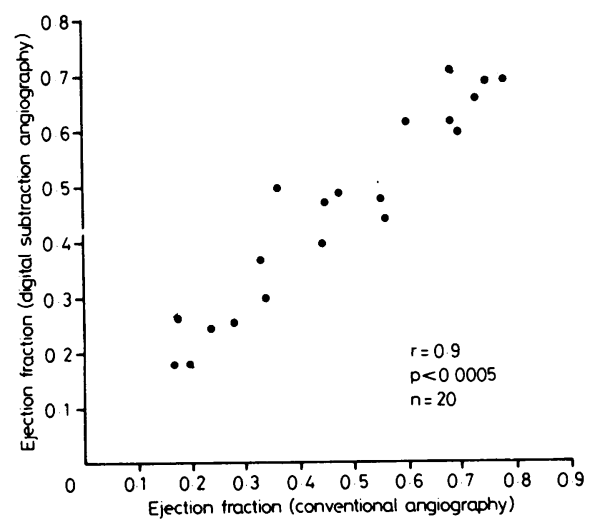

Fig. 2 Ejection fraction by digital subtraction angiography plotted against ejection fraction by conventional angiography. The broken lines indicate $95 \%$ confidence limits. Correlation coefficient $r=0.9, p<0.0005, n=20$. native technique of using an injection of small volumes of contrast medium directly into the left ventricle rather than larger intravenous volumes. The practicality of this approach has been investigated in patients with good left ventricular function by computer assisted post-processing of images recorded on conventional cinefilm. ${ }^{9}$ Using a prototype apparatus with an effective framing rate of 10 frames/s and diluted contrast medium a close correlation was found for ejection fractions estimated by both digital and conventional techniques. ${ }^{10}$ Another group using low volume intraventricular injections confirmed the close correlation and also noted that digital subtraction successfully identified left ventricular wall motion abnormalities. ${ }^{11}$

Many of these studies have used modifications to conventional $x$ ray apparatus or prototype machines that are not generally available. In the present study we used a dedicated, commercially available system that, unlike many others, has the advantage of storing the data in digital rather than analogue form. The results underline the important advantage over conventional angiography of providing images that are free of extrasystoles. Previous workers have generally studied patients with ejection fractions greater than 0.5 . Indeed, one worker ${ }^{12}$ considered that digital subtraction was likely to yield poor results when applied to patients with more severe impairment of left ventricular function.

The present study confirms that after the intravenous injection of contrast medium left ventricular wall movement is reliably demonstrated by digital subtraction angiography. Thus patients with globally impaired wall movement can be distinguished from those with a localised abnormality such as an aneurysm. Diagnostic images were obtained even in patients with considerably reduced ejection fractions. Patients with a potentially surgically correctable lesion may, therefore, be distinguished from those with a severe generalised impairment of function that is not amenable to surgery. The patient may be spared the inconvenience of hospital admission and arterial puncture. Digital subtraction, independent of the availability of a radioisotope, has advantages over nuclear ventriculography. Unlike many nuclear studies it is a "first pass" technique.

The pixel density used $(256 \times 256)$ affords much better resolution than that of nuclear medicine $(64 \times$ 64). At present, temporal resolution is inferior to that provided by some first pass nuclear studies, but improvements in computer software are likely to increase the framing rate considerably. As with both conventional and nuclear angiography heart valves are not defined. At present, the demonstration of coronary artery anatomy after intravenous injection of contrast medium has been unsuccessful. 

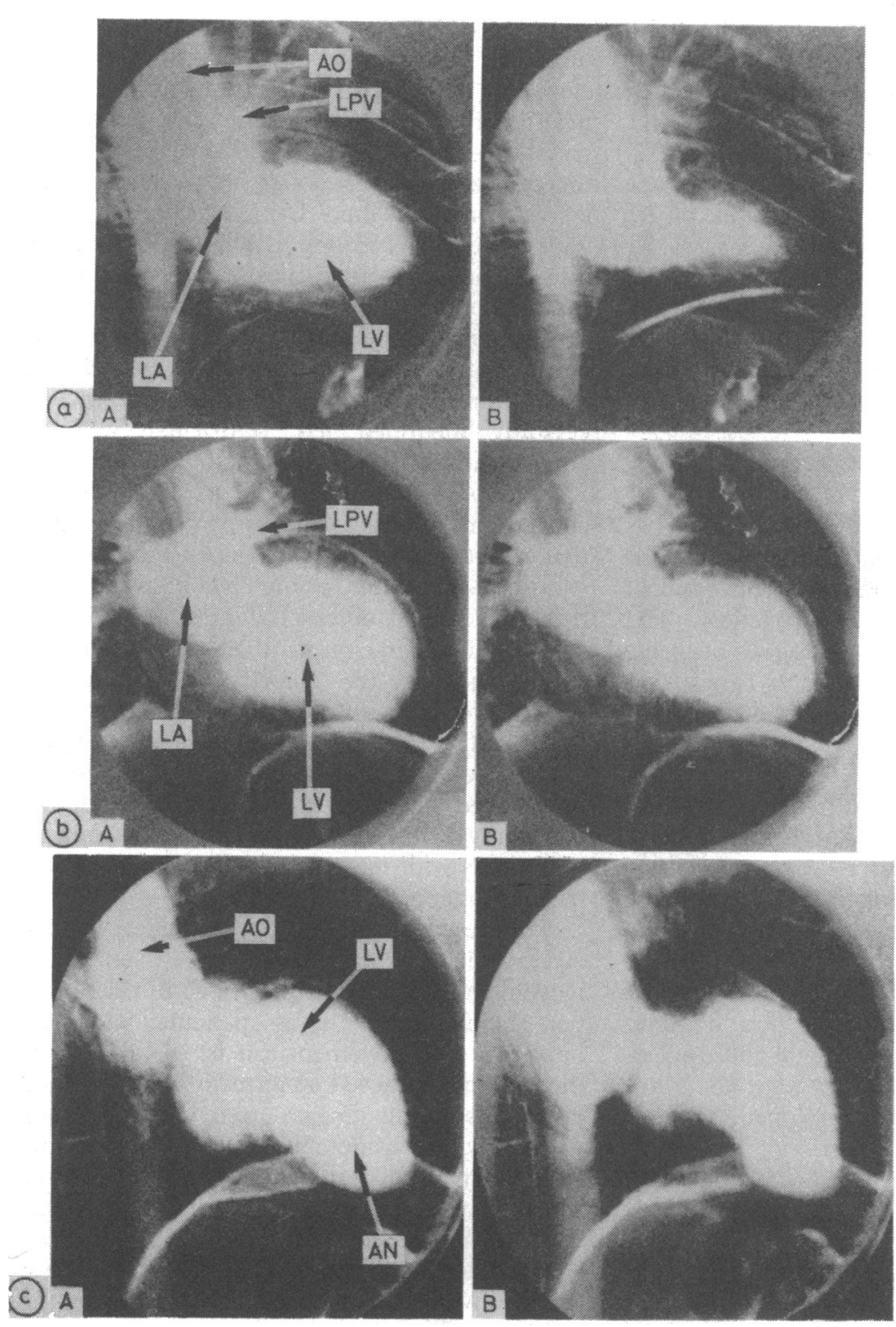

Fig. 3 Digital angiograms showing end diastolic $(A)$ and end systolic ( $B$ ) frames in (a) a normal subject; (b) a patient with generalised impairment of left ventricular wall movement and no discrete aneurysm; and (c) $a$ patient with an apical aneurysm. $A O$, aorta; $L P V$, left pulmonary vein; $L A$, left atrium; $L V$, left ventricle; $A N$, aneurysm.

\section{CONCLUSION}

Digital subtraction angiography is a relatively simple and convenient method of visualising the left ventricle. Although its precise role in the investigation of patients has yet to be defined, this study suggests that it provides an accurate assessment of ejection fraction. It also distinguishes patients with generalised impairment of left ventricular function from those with a potentially surgically correctable defect. Because it is relatively less invasive this technique appears to offer the prospect of a better understanding of the progression of left ventricular disease as serial studies may be possible.

We thank the London Imaging Centre for allowing the use of facilities that made this study possible. We also thank Dr C Symons for allowing patients under his care to be included in this study.

\section{References}

1 Mistretta CA, Crummy AB, Strother CM. Digital angiography: a perspective. Radiology 1981; 139: 273-6. 
2 Dodge HT. Determination of left ventricular volume and mass. Radiol Clin North Am 1971; 9: 459-67.

3 Robb GP, Steinberg I. Visualisation of the chambers of the heart and pulmonary circulation and the great blood vessels in man. $A \mathcal{R}$ 1939; 41: 1-17.

4 Kruger RA, Mistretta CA, Houk TK, et al. Computerized fluoroscopy in real time for noninvasive visualization of the cardiovascular system. Radiology 1979; 130: 49 57.

5 Meaney TF, Weinstein MA, Buonocore E, et al. Digital subtraction angiography of the human cardiovascular system. AfR 1980; 135: 1153-60.

6 Ovitt TW. Intravenous angiography utilizing digital video subtraction techniques. Am $\mathcal{F}$ Cardiol 1982; 49: 1365-7.

7 Engels PHC, Ludwig JW, Verhoeven LAJ. Left ventricle evaluation by digital video subtraction angiocardiography. Radiology 1982; 144: 471-4.
8 Higgins CB, Norris SL, Gerber KH, Slutsky RA, Ashburn WL, Baily N. Quantitation of left ventricular dimensions and function by digital video subtraction angiography. Radiology 1982; 144: 461-9.

9 Sasayama S, Nonogi H, Kawai C, Fujita M, Eiho S, Kuwahara M. Automated method for left ventricular volume measurement by cine ventriculography with minimal doses of contrast medium. Am $\mathcal{F}$ Cardiol 1981; 48: 746-53.

10 Nichols AB, Martin EC, Fles TP, et al. Validation of the angiographic accuracy of digital left ventriculography. Am f Cardiol 1983; 51: 224-30.

11 Tobis JM, Nalcioglu O, Johnstone WD, et al. Correlation of 10-milliliter digital subtraction ventriculograms compared with standard cineangiograms. Am Heart $\mathcal{f}$ 1983; 105: 946-52.

12 Raphael M. Digital subtraction angiography. Current medical literature. Cardiovascular Medicine 1983; 2: 5-7. 